\section{Meta-research in geriatric medicine: a survey of the Italian Society of Hospital and Community Geriatrics (SIGOT)}

\begin{abstract}
Nicola Veronese, ${ }^{1,2}$ Jacopo Demurtas, ${ }^{3}$ Stefania Maggi, ${ }^{4}$ Antonio Cherubini, ${ }^{5}$ Vincenzo Solfrizzi, ${ }^{6}$ Alberto Cella, ${ }^{7}$ Filippo Luca Fimognari, ${ }^{8}$

Alberto Ferrari, ${ }^{9}$ Alberto Pilotto ${ }^{7,10}$

${ }^{1}$ Geriatric Unit, Department of Internal Medicine and Geriatrics, University of Palermo, Palermo; ${ }^{2}$ Primary Care Department, Azienda ULSS 3 (Unità Locale Socio Sanitaria) Serenissima, Dolo-Mirano District, Venice; ${ }^{3}$ Primary research for clinical practice and almost all the participants recognized that metaresearch has changed their daily clinical approach to the patient. Almost all the participants would like to have more training in meta-research suggesting that SIGOT should organize training courses for metaresearch in geriatric medicine. In conclusion, our survey showed that the interest in SRs/MAs is high, whilst the knowledge is still limited suggesting that education is needed to fill the gap in this field.
\end{abstract} Care Department, Azienda USL Toscana Sud Est, Grosseto; ${ }^{4}$ Consiglio Nazionale delle Ricerche, Neuroscience

Department, Aging Branch, Padova; ${ }^{5}$ Geriatria, Accettazione Geriatrica e Centro di ricerca per l'invecchiamento, IRCCS INRCA, Ancona; ${ }^{6} \mathrm{C}$. Frugoni Internal and Geriatric Medicine and Memory Unit, University of Bari Aldo Moro, Bari; 'Department of Geriatric Care, Orthogeriatrics and

Rehabilitation, EO Galliera Hospital, Genova; ${ }^{8}$ Unit of Geriatrics, Cosenza Hospital, Cosenza; ${ }^{9} 3 \mathrm{C}$ Salute - Impresa Sociale scs, Reggio Emilia; ${ }^{10}$ Department of Interdisciplinary Medicine, University of Bari, Bari, Italy

\footnotetext{
Abstract

The need for major information in meta-research (i.e. the part of medicine interested in systematic reviews [SRs] and meta-analyses [MAs]) is increasing. In the last years we are observing an exponential rate of publications as SRs/MAs in geriatric medicine. In order to better assess the interest in meta-research, we proposed a survey to know the knowledge and the needs in meta-research in geriatrics. A short survey (about 5 minutes) was freely available in the Italian Society of Hospital and Community Geriatrics (SIGOT) website and diffused in social networks. The survey was available during the entire 2019. The survey regards demographic information, previous research activities and the knowledge of the participant on meta-research. Altogether, 148 participants mainly men $(=65.5 \%)$ and mainly aged 36 to 44 years and working in hospital from all Italian regions completed the survey. Responders
}

read more than 20 articles in the previous year $(=58.1 \%)$, including $10-20$ SRs/MAs (25\%). Many respondents (41.9\%) had published $>20$ articles during their life. At the same time, a consistent part of the respondents recognized the importance of meta-

\section{Introduction}

Systematic reviews and meta-analyses (SRs and MAs), the process of synthesizing previously published research evidence proposed for answering specific questions in clinical practice, are commonly believed as the highest evidence in the scientific pyramid. ${ }^{1}$ It is widely known that SRs and MAs can significantly contribute to clinical practice, since they can increase knowledge and identify fields, where current evidence is still lacking. Unfortunately, many SRs and MAs have been considered redundant, misleading, serving conflicting interests or of low-quality. These shortcomings, instead improving actual evidence, multiply the limitations of primary studies, rather than objectively and critically presenting them. ${ }^{2,3}$ These issues are widely applicable to geriatric medicine. ${ }^{4}$

Physicians and other people working in medicine need to know recent developments within their professional area. Consequently, there is need for articles that summarize available knowledge by using systematic processes. In our opinion, to decide whether a research article is of high quality and therefore trustworthily, potential readers need to know the key issues of such research and article types.

Geriatric medicine has some specific requirements that an investigator should considering when approaching a SR and/or MA. ${ }^{4}$ The accurate identification/inclusion of specific groups (such as older persons pertinent to daily practice) is crucial as well as the high drop-out rate of older people observed in several randomized controlled trials (RCTs). In addition, the low reporting of older people in RCTs is another important shortcoming. ${ }^{4-6}$ Finally, to the best of our knowledge, little is known about the
Correspondence: Nicola Veronese, Geriatric Unit, Department of Internal Medicine and Geriatrics, University of Palermo, viale F. Scaduto 6/c, 90144 Palermo, Italy.

E-mail: nicola.veronese@unipa.it

Key words: Meta-analysis; systematic review; questionnaire; SIGOT.

Contributions: NV, JD, AP, prepared the survey and wrote the first draft of the paper; $\mathrm{SM}, \mathrm{AC} 1$, VS, FLF, AF, critically revised the paper; $\mathrm{AC} 2$, data analysis.

Conflict of interests: the authors declare no potential conflict of interests.

Availability of data and material: all the data are available upon request to the corresponding author.

Ethics approval: not applicable.

Consent to participate: not applicable.

Consent for publication: not applicable.

Received for publication: 5 September 2020. Revision received: 14 October 2020

Accepted for publication: 14 October 2020

This work is licensed under a Creative Commons Attribution-NonCommercial 4.0 International License (CC BY-NC 4.0).

${ }^{\circ}$ Copyright: the Author(s), 2020

Licensee PAGEPress, Italy

Geriatric Care 2020; 6:9339

doi:10.4081/gc.2020.9339

interest and competence of physicians interested in older people care regarding SRs and MAs. Only recently, the European Geriatric Medicine Society (EuGMS) has reported some findings regarding the knowledge in meta-research across their members, ${ }^{7}$ but the specific knowledge on meta-research among Italian researchers is still not known.

Given this background, to have information clinicians and researchers interested in geriatric medicine and working in Italy, we assessed the scientific background, knowledge and opinions regarding meta-research in geriatric medicine through an online survey, using the network and membership tools of the Italian Society of Hospital and Community Geriatrics (SIGOT).

\section{Materials and Methods}

The questionnaire was formulated and approved by the Scientific Committee of the 
SIGOT. The data were fully anonymized and informed consent was provided by all participants during the questionnaire.

We reported the findings of the survey according to the reporting guidelines for survey research. ${ }^{8}$ Two authors (NV, JD) designed an online survey for obtaining information regarding the knowledge and interest of people involved in SIGOT activities. The survey, in Italian, was proposed using Google Forms and was sent out in January 2019 by the SIGOT secretariat to all SIGOT members. An invitation for the survey to all members by email and with the monthly newsletter. Finally, the survey was advertised during the oral presentation Attività di ricerca SIGOT: update 2019 during the $33^{\text {th }}$ SIGOT congress in Bologna (57 May 2019).

The full questionnaire was available online as link at the SIGOT homepage (https://www.sigot.org/), and stayed online from $06^{\text {th }}$ January until $31^{\text {st }}$ December 2019.

Questions were structured under three principal headings: general characteristics/ demographics, scientific background, knowledge and opinions regarding meta-research. All the questions were mandatory. The survey took about 5 minutes for being completed.

All categorical variables were reported descriptively as percentages (\%) relative to the total number of participants included.

\section{Results}

Overall, 148 participants from all Italian regions responded to the survey. Table 1 summarizes the questions and the most common answer for each question. A higher prevalence of males $(65.5 \%)$ was observed and young respondents (36-44 years) were the most represented across all age groups (19.6\%). The majority of the respondents were geriatricians $(70.3 \%)$, worked in hospitals $(64.2 \%)$ and had more than 20 years from specialization $(41.6 \%)$.

The majority of participants $(58.1 \%)$ had read more than 20 scientific articles in the previous year, including 10-20 SRs/MAs $(25.0 \%)$. The majority of the respondents $(41.9 \%)$ had published $>20$ articles during their life.

Finally, in the last part, we assessed the participants' knowledge regarding metaresearch. First, respondents reported that SRs and/or MAs are extremely important in their clinical practice $(36.5 \%$ gave a score of 8 , in a score between 0 and 10). However, the majority of the participants reported a lack of expertise in metaresearch $(49.3 \%$ gave $<5 / 10)$. A relevant need for education on reading and interpreting SRs and MAs ( 8 and 9/10 were chosen by the $20.9 \%$ of the participants, respectively) was evident. Residential courses were the preferred way to learn $(39.9 \%)$, followed by to follow an expert $(29.7 \%)$.

Finally, we also asked to judge the importance of SRs and MAs. Almost all participants indicated that SRs/MAs had significantly changed their clinical approach to their patients. Regarding the quality of SRs and MAs, in the field of geriatrics, the participants indicated that the scientific quality is good $(29.1 \%$ said that the quality of these publications is $8 / 10$ ). Of the 148 participants, $29.6 \%$ had written a systematic review/meta-analysis themselves and they were mainly involved in the first steps of this type of research, i.e. biblio- graphic search or data extraction. Finally, a large majority of the respondents did not write a SR/MA during their life (81.8\%).

\section{Discussion}

This survey, including 148 participants from all Italian regions, shows their knowledge, opinions and preferences regarding meta-research applied to geriatric medicine.

The respondents to this survey were mainly young geriatricians, working in hospital with a good academic engagement. A consistent part read several scientific articles during the previous year $(>20)$ and had published several scientific articles during their life, although the reading of SRs and MAs was limited to a small number.

It should be noted that survey participants are often those scientifically more active and engaged in research. ${ }^{7}$ Therefore, the participants should represent those who more often consult and read the actual literature. These participants, as also previously shown, ${ }^{7}$ strongly think that meta-research is of clinical importance, since has significantly changed the clinical approach to their patients. In this sense, the respondents reported that more education in SRs/MAs is needed, suggesting that the SIGOT should organize specific courses in this regard.

Finally, in the last part of our survey, systematic reviews and meta-analyses are considered of clinical importance, probably because they synthesize the (published) scientific knowledge of original studies regarding a specific research question. About a quarter of the interviewed participants believe that the actual systematic

Table 1. Main findings of the survey regarding meta-research.

1.4 Years from specialization

1.5 City (region)

1.6 Workplace

$\begin{array}{cc}\text { Male } & 65.5 \\ \text { 36-44 years } & 19.6 \\ \text { Geriatrics } & 70.3 \\ >20 & 41.6 \\ \text { Genoa (Italy) } & 11.5 \\ \text { Hospital } & 64.2 \\ >20 & 58.1 \\ 10-20 & 25.0 \\ >20 & 41.9 \\ 8 & 36.5 \\ 6 & 22.3 \\ 8 \text { or } 9 & 20.9 \\ \text { To follow residential courses } & 39.9 \\ \text { Yes } & 94.4 \\ \text { Yes } & 84.5 \\ 8 & 29.1 \\ \text { No } & 81.8\end{array}$


reviews and meta-analyses in geriatric medicine are of good quality.

This work has some strengths that we would like to mention. For example, the sample size included is large and representative of all Italian territory. Some shortcomings should be mentioned. First, there was a preponderance of early/mid-career researchers, and the perspectives given may not be representative of more junior or senior colleagues. Therefore, it is possible that a selection bias exists, i.e. those who were more active readers or researchers may had been more likely to complete the survey, but it is a common limitation in online surveys. Finally, since participants were asked information regarding the previous year, a recall bias might also be present.

\section{Conclusions}

In conclusion, our survey showed that interest in meta-research in the SIGOT is a relevant need, whilst knowledge is limited, suggesting that more education, particularly through residential courses, is needed. Courses specific for geriatric medicine topics and tailored for different degrees in knowledge in meta-research are therefore needed for satisfying this necessity.

\section{References}

1. Stevens KR. Systematic reviews: the heart of evidence-based practice. AACN Advanced Critical Care 2001;12:529-38.

2. Møller MH, Ioannidis JP, Darmon M. Are systematic reviews and meta-analyses still useful research? We are not sure. Berlin: Springer; 2018.

3. Ioannidis JP, Lau J. Pooling research results: benefits and limitations of meta-analysis. Joint Commission J Qual Improv 1999;25:462-9.
4. Shenkin SD, Harrison JK, Wilkinson $\mathrm{T}$, et al. Systematic reviews: guidance relevant for studies of older people. Age Ageing 2017;46:722-8.

5. Cherubini A, Signore SD, Ouslander J, et al. Fighting against age discrimination in clinical trials. J Am Geriatr Soc 2010;58:1791-6.

6. Crome P, Cherubini A, Oristrell J. The PREDICT (increasing the participation of the elderly in clinical trials) study: the charter and beyond. Expert Rev Clin Pharmacol 2014;7:457-68.

7. Veronese N, Torbahn G, Demurtas J, et al. Interest in meta-research in geriatric medicine: a survey of members of the European Geriatric Medicine Society. Eur Geriatr Med 2020:1-5.

8. Kelley K, Clark B, Brown V, Sitzia J. Good practice in the conduct and reporting of survey research. Int J Qual Health Care 2003;15:261-6. 